\title{
Schistosomiasis mansoni is Asssociated with Pyogenic Liver Abscesses in the State of Minas Gerais, Brazil
}

\author{
Rosângela Teixeira/ ${ }^{+}$, Francis Jardim Pfeilsticker, Giovanna DC Santa Cecília, \\ Vandack Nobre, Lúcia Porto Fonseca*, José Carlos Serufo, Paulo Marcos Zech \\ Coelho**, José Roberto Lambertucci
}

\author{
Departamento de Clínica Médica *Anatomia Patológica, Faculdade de Medicina **Departamento de \\ Parasitologia, ICB/UFMG e Santa Casa de Misericórdia, Avenida Alfredo Balena 190, 4º andar, 30130-100 \\ Belo Horizonte, MG, Brasil
}

The association between pyogenic liver abscesses and schistosomiasis has been confirmed by clinical and experimental studies. In this retrospective study of 78 patients with pyogenic liver abscesses the association with schistosomiasis has been investigated. Pyodermitis, a known focus of bacteremia, was observed in 19 patients (24\%). Blood eosinophilia was observed in 30 patients (39\%). Staphylococcus aureus was cultured from abscesses in 17 out of 38 patients (45\%). Forty-one out of 57 patients (53\%) had stool examination. Schistosoma mansoni was the main parasite identified. Eggs of S. mansoni were also identified in liver biopsies in 7 out of 19 patients who did the exam. The large number of young patients with liver abscesses described here is different from what has been observed in developed countries. This clinical study provide support for the concept that granulomas of S. mansoni in the liver are foci for colonization with S. aureus, which in presence of staphylococcal bacteremia can form liver abscesses.

Key words: liver abscess - schistosomiasis - staphylococcal bacteremia - Minas Gerais - Brazil

Pyogenic liver abscesses are relatively uncommon, despite the frequency of cholecystitis, appendicitis and diverticulitis, which are the main sources of bacterial infection to the liver. In adults, the abscesses are frequently polymicrobial, usually due to enteric Gram-negative bacilli (e.g. Escherichia coli) and anaerobic bacteria. In general, Staphylococcus aureus liver abscesses are associated with infection in other organs, abdominal trauma or immunosuppressive diseases in children up to 5 years of age (Moss \& Pyscher 1981) or related to thrombophlebitis of the umbilical vein.

In South America, parasitic diseases have been described as a predisposing factor for pyogenic liver abscesses. Amoebic liver abscesses (Barnes et al. 1987) as well as ecchinococcus hepatic cyst (Levinson \& Bush 1995) may be secondarily infected with bacteria. In addition, migrating Ascaris lumbricoides (Salviano Filho et al. 1973, Rossi \& Bisson 1983) and, more recently, acute schistoso-

\footnotetext{
${ }^{+}$Corresponding author. Fax: +55-31-3344.6625. E-mail: teixeira@medicina.ufmg.br

Received 14 May 2001

Accepted 25 July 2001
}

miasis (Lambertucci et al. 1990, 1997, Teixeira et al. 1996) have been implicated as predisposing factors in the genesis of pyogenic liver abscesses.

We previously reported a clinical and experimental study of the association between schistosomiasis and pyogenic liver abscess. Our study demonstrated that acute schistosomiasis concurrent with $S$. aureus bacteremia favors the colonization of the liver by bacteria causing pyogenic hepatic abscesses schistosomiasis (Lambertucci et al. 1990, 1997, Teixeira et al. 1996).

This study sought to investigate whether liver abscesses in our state are associated with schistosomiasis mansoni, in a retrospective evaluation of 78 patients with pyogenic liver abscesses.

\section{PATIENTS AND METHODS}

Seventy-eight patients with liver abscesses admitted to five general hospitals of Belo Horizonte, State of Minas Gerais, Brazil (MG), from 1981 to 1997, had their medical charts selected for the study. Clinical, epidemiological and laboratory data were reviewed in order to complete a standardized questionnaire further analyzed in a database bank (EpiInfo v. 6.0).

All patients came from MG, an endemic area of schistosomiasis mansoni. Fifty-five patients were male (73\%), with ages ranging from 3 to 75 years (mean 25, median: 16) (Table). 
TABLE

Main characteristics of 78 patients with pyogenic liver abscesses

\begin{tabular}{lcc}
\hline Patients' characteristics & $\begin{array}{c}\text { Total } \\
(\mathrm{n}=78)\end{array}$ & $\%$ \\
\hline Sex (male/female) & $57 / 21$ & $73 / 27$ \\
Age (years) & 3 to 75 & \\
3 to 12 & 27 & 35 \\
13 to 20 & 16 & 21 \\
21 to 75 & 34 & 44 \\
Clinical symptoms & & \\
Fever & 58 & 75 \\
Hepatomegaly & 58 & 75 \\
Abdominal pain & 68 & 87 \\
Splenomegaly & 7 & 9 \\
Pyodermitis & 19 & 24 \\
\hline
\end{tabular}

On admission, all patients appeared ill and were submitted to clinical examination. The most important symptoms of patients are presented in the Table. The following subjacent diseases were disclosed: malnutrition $(n=5)$, alcoholic abuse $(n=5)$, lytiasis billiar $(n=4)$, previous abdominal surgery $(\mathrm{n}=3)$, diabetes mellitus $(\mathrm{n}=2)$, neoplasic diseases $(\mathrm{n}=2)$, ascaridiasis $(\mathrm{n}=2)$, acute pancreatitis $(\mathrm{n}=$ $1)$, and billiar fistulae $(n=1)$. Pyodermitis, a known focus of bacteremia, was noted clinically in 19 patients $(24 \%)$.

Laboratory analysis included white and red blood cells count $(n=78)$, parasitological stool examination (Lutz, HPJ method) $(\mathrm{n}=41)$, blood culture $(n=31)$ and culture of purulent secretion taken from liver abscesses $(\mathrm{n}=38)$. Investigations also included abdominal ultrasonography $(\mathrm{n}=58)$, abdominal CT-scan ( $\mathrm{n}=23$ patients) and liver biopsies $(n=19)$. One patient was submitted to rectal biopsy.

All patients were submitted to supportive and antimicrobial treatment. Surgical drainage of the abscesses was performed in 31 patients. Nine patients were submitted to percutaneous drainage only. Patients with schistosomiasis were treated with oxamniquine after clinical improvement. All patients recovered.

\section{RESULTS}

Laboratory analysis showed blood eosinophilia (peripheral blood absolute eosinophils count $>400$ ) in 30 patients (39\%). Forty-one patients did at least one parasitological stool examination. The following parasites were identified: $S$. mansoni in 22 patients $(65 \%)$, A. lumbricoides in 9 (26\%), Tricocephalus trichiuris in $5(15 \%)$, Ancylostoma duodenale in 3 (9\%), Strongyloides stercoralis in
$4(12 \%)$, Necator americanus in $2(6 \%)$, and Hymenolepis nana in $2(6 \%)$. Nineteen patients (24\%) presented co-infected with $S$. mansoni and one of the above parasites.

The blood culture was positive in $10(32 \%)$ out of 31 patients. $S$. aureus was isolated from blood culture of 5 patients (50\%), and Gram-negative bacteria in the others.

The culture of purulent secretion from liver abscesses performed in 38 patients was positive in 35 $(92 \%)$. S. aureus was the single bacteria isolated in 17 cases $(45 \%)$, S. pyogenes in 9 cases (23\%), Streptococcus sp. in 4 (10\%), Gram-negative in $2(5 \%)$. Two bacteria were isolated in liver abscesses from $3(8 \%)$ patients and 3 cultures $(8 \%)$ resulted negative.

The diagnosis of pyogenic liver abscess was confirmed by ultrasonography $(\mathrm{n}=53)$, and abdominal CT-scan $(n=23)$. Six patients presented periportal thickening in ultrasonography.

The diagnosis of schistosomiasis was confirmed by liver biopsies in 7 (37\%) out of 19 patients (Fig. 1). Six out of 7 patients also presented eggs of $S$. mansoni in the stool examination, and one patient had the diagnosis of schistosomiasis confirmed by rectal biopsy.

To illustrate the clinical presentation and evolution of a patient with the association of pyogenic liver abscess and schistosomiasis, one of our cases has been selected and summarized below.

Case report - A 5 years old boy was admitted to hospital because of high fever, malaise, pain on the right side of the abdomen, and diarrhea that started 8 days before. Physical examination revealed skin pallor, tachypnea and hepatosplenomegaly. A laboratory make up showed hematocrit of $30 \%$, leukocytosis and eosinophilia. S. mansoni eggs were identified in the stools. Abdominal ultrasound followed by a CT-scan revealed a huge abscess in the left lobe of the liver (Fig. 2). Treatment was started with oxacillin and amikacin, but no convincing clinical response was observed. Ten days later he was operated on with drainage of the abscess. Liver biopsy taken on the occasion showed a great number of $S$. mansoni eggs in the fragments. S. aureus was isolated from the purulent material obtained during surgery. Treatment with oxacillin was maintained for another three weeks with progressive improvement and the patient was discharged from hospital in a good clinical condition.

\section{DISCUSSION}

Our results demonstrated a high prevalence of patients with liver abscesses and with schistosomiasis mansoni. This association has not been observed in developed countries. Although we have 


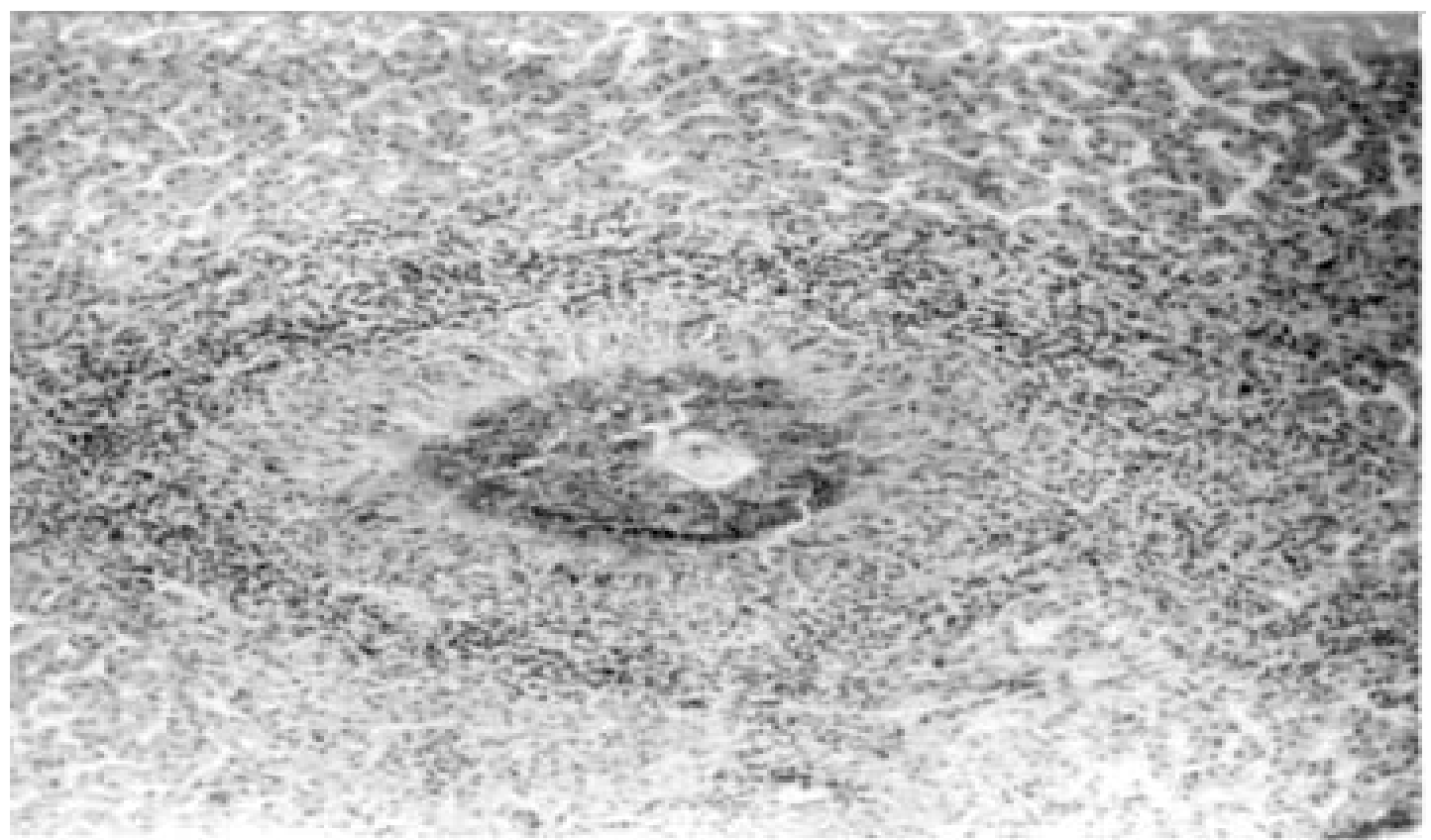

Fig. 1: section through a liver biopsy showing a recent granuloma of Schistosoma mansoni around a miracidium-containg egg (center) (Haematoxilin and eosin staining, x 200).

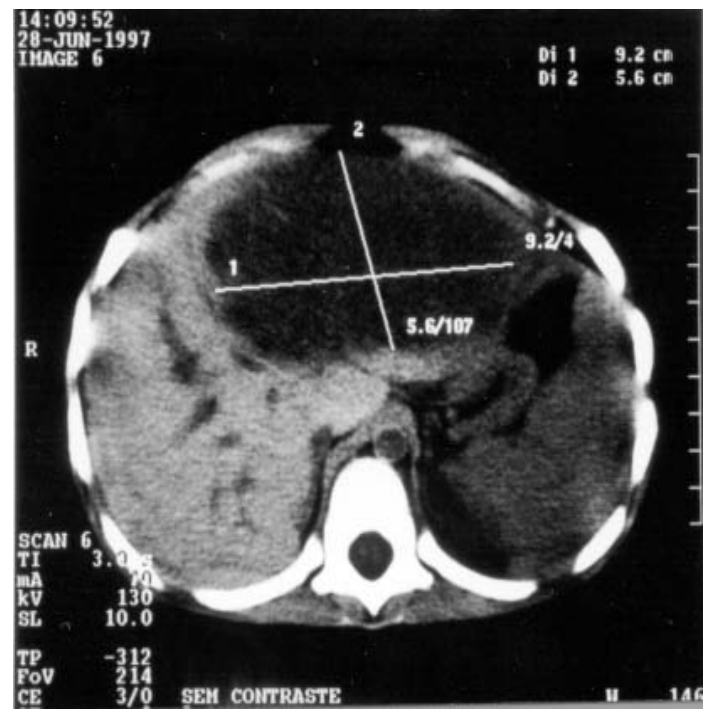

Fig. 2: CT-scan showing a huge abscess in the left lobe of the liver.

found a high prevalence of co-infection with other parasitic diseases, S. mansoni was the main parasite identified in patients with pyogenic liver abscesses in MG. In addition, blood eosinophilia, which is uncommon in the course of sepsis, was found in a large number of patients and suggest a possible association of liver abscesses with parasitic diseases.

Although the investigation of the molecular basis of the association between schistosomiasis mansoni and pyogenic liver abscesses has not been described yet, the clinical data presented here, together with the results of our previous experimental study (Teixeira et al. 1996) suggest that the adhesion of S. aureus to granulomata of S. mansoni is implicated in the pathogenesis of this association.

Several pathogenic mechanisms have been proposed to explain this provocative association. Studies have demonstrated that the crucial event that starts an infection is the adherence of the microorganism to the cells of the host. This complex phenomenon involves both inflammatory cells and the microorganisms (Reed \& Williams 1983). It has been demonstrated that Gram-positive organisms avidly bind to matrix-protein-coated surfaces, and $S$. aureus, in particular, aggregates in the presence of fibronectin, laminin, and type IV collagen (Vercellotti et al. 1985). As for collagen-associated proteoglycans, fibronectin is abundant during the more active stages of the granuloma (Andrade 1991). This suggests that $S$. aureus might adhere to compounds of the extracellular matrix of the granulomata of S. mansoni, e.g. collagen, fibronectin and proteoglycans (Andrade 1991), and, consequently, liver abscesses can be formed. In addition, the formation and degradation of extracellular matrix and laminin of the chronic granuloma (Andrade \& Grimaud 1988) may also be implicated in the pathogenesis of the abscesses. 
Besides a local adhesion mechanism, the immune response in schistosomiasis infection may play a role in the pathogenesis of this association. The inhibition of Th1 immune response noted after the deposition of eggs (Pearce et al. 1991, Grzych et al. 1991) can affect the normal response against bacterial infection. The eosinophilia and the hyperimmunoglobulinaemia $\mathrm{E}$ that occur in acute schistosomiasis might reduce host defenses against bacteria, similar to other syndromes, where great increase in IgE is associated with undue susceptibility to infection (Buckley et al. 1972, Lambertucci 1998). Additionally, the neutropenia that occurs in some periods of schistosomiasis infection (Lenzi et al. 1995) may play a role in the pathogenesis of this association. Finally, degenerated worms colonized by $S$. aureus lodged in the hepatic parenchyma, as previously demonstrated in our study (Teixeira et al. 1996), might be implicated in the pathogenesis of the liver abscesses, as already showed with other worms (e.g. A. lumbricoides) lodged in the liver (Salviano Filho et al. 1973, Rossi \& Bisson 1983).

In short, this clinical study and our previous experimental study provide support for the concept that eggs and granulomas of S. mansoni in the liver portal triads are foci for colonization with S. aureus, which in the presence of a staphylococcal bacteremia can form pyogenic liver abscesses. More intensive clinical investigation in endemic areas of schistosomiasis and studies of the molecular basis of these diseases may lead to further understanding of the association.

\section{REFERENCES}

Andrade ZA 1991. Extracelular matrix and schistosomiasis. Mem Inst Oswaldo Cruz 86: 61-73.

Andrade ZA, Grimaud JA 1988. Morphology of chronic collagen reabsortion. A study on the late stages of schistosomal granuloma involution. Am J Pathol 132: 389-399.

Barnes PF, Kevin M, De Cock KM, Reynolds TN, Ralls PW 1987. A comparison of amoebic and pyogenic abscess of the liver. Medicine 66: 472-483.

Buckley RH, Wray BB, Belmaker EZ 1972. Extreme hyperimmunoglobulinemia $\mathrm{E}$ and undue susceptibility to infection. Pediatrics 49: 57-70.
Grzych JM, Pearce E, Cheever A, Caulada ZA, Caspar P, Heiny S, Lewis S, Sher A 1991. Egg deposition is the major stimulus for the production of the Th2 cytokines in murine schistosomiasis mansoni. $J$ Immunol 146: 1322-1327.

Lambertucci JR 1998. Hyperimmunoglobulinemia E, parasitic diseases and staphylococal infection. Rev Soc Bras Med Trop 29: 407-410.

Lambertucci JR, Rayes AAM, Barata CH, Teixeira R, Gerspacher-Lara R 1997. Acute schistosomiasis: report on five singular cases. Mem Inst Oswaldo Cruz, 92: 631-635.

Lambertucci JR, Teixeira R, Navarro MMM, Coelho PMZ, Ferreira MD 1990. Liver abscess and schistosomiasis; a new association. Rev Soc Bras Med Trop 23: 239-240.

Lenzi HL, Lenzi JA, Rosman FC, Pelajo-Machado M, Mota EM, Panasco MS, Oliveira DN 1995. Extramedullary hematopoiesis in murine schistosomiasis mansoni. Mem Inst Oswaldo Cruz 90: 169-177.

Levinson ME, Bush LM 1995. Peritonitis and other intra-abdominal infections. In GL Mandell, JE Bennett (eds), Principles and Practice of Infectious Diseases, Churchill Livingstone, New York, p. 705-740.

Moss TJ, Pysher TJ 1981. Hepatic abscess in neonates. Am J Dis Children 135: 726-728.

Pearce EJ, Caspar P, Grzych JM, Lewis FA, Sher A 1991. Dowregulation of Th1 cytokine production accompanies induction of $\mathrm{Th} 2$ responses by a parasitic helmint Schistosoma mansoni. J Exper Med 173: 159-166.

Reed NW, Williams RC 1983. Bacterial adherence: first step in the pathogenesis of certain infections. $J$ Chronic Dis 31: 67-92.

Rossi M, Bisson FW 1983. Fatal case of multiple liver abscesses caused by adult Ascaris lumbricoides. Am J Trop Med Hyg 32: 523-525.

Salviano Filho P, Kalume RS, Andrade JCC 1973. Abscesso hepático; experiência do HSE em 25 anos, revisão da literatura brasileira. Rev Méd HSE 25: 299-331.

Teixeira R, Ferreira MD, Coelho PMZ, Brasileiro Filho G, Azevedo Júnior GM, Lambertucci JR 1996. Pyogenic liver abscesses and acute schistosomiasis mansoni: report on 3 cases and experimental study. Trans $R$ Soc Trop Med Hyg 90: 280-283.

Vercellotti GM, Mc Carty JB, Lindholm P, Peterson PK, Jacob HS, Furcht LT 1985. Extracellular matrix proteins (fibronectin, laminin and type IV collagen) bind and aggregate bacteria. Am J Pathol 120: 13-21. 